\title{
Erratum: Double-peaked inflation model: Scalar induced gravitational waves and primordial-black-hole suppression from primordial non-Gaussianity [Phys. Rev. D 104, 063515 (2021)]
}

Fengge Zhang (张丰阁), Jiong Lin (林昫), and Yizhou Lu (卢一洲)

(ه) (Received 2 December 2021; published 21 December 2021)

DOI: $10.1103 /$ PhysRevD.104.129902

A minus sign in Eq. (26) was missing in our paper. The right relationship between primordial curvature perturbation and the density contrast is

$$
\delta(\boldsymbol{x})=-\frac{4}{9}\left(\frac{1}{a H}\right)^{2} \nabla^{2} \zeta(\boldsymbol{x})
$$

or that in Fourier space $[1,2]$

$$
\delta_{k}=\frac{4}{9}\left(\frac{k}{a H}\right)^{2} \zeta_{k} .
$$

Then there are no minus signs in Eqs. (32), (33), (34), and (35). As a result, a positive non-Gaussianity parameter $f_{\mathrm{NL}}$ boosts the primordial black holes (PBHs) formation, while on the contrary, a negative one makes PBHs form hardly. So the abundance of PBHs should be enhanced rather than suppressed in our model. But the other conclusions remain.

[1] A. M. Green, A. R. Liddle, K. A. Malik, and M. Sasaki, Phys. Rev. D 70, 041502 (2004).

[2] A. R. Liddle and D. H. Lyth, Cosmological Inflation and Large-Scale Structure (Cambridge University Press, Cambridge, England, 2000). 